\title{
Relationship between fiber degradation and residence time distribution in the processing of long fiber reinforced thermoplastics
}

\author{
H. Zhuang*, P. Ren, Y. Zong, G. C. Dai \\ State Key Laboratory of Chemical Engineering, East China University of Science and Technology, \\ 200237 Shanghai, China
}

Received 14 May 2008; accepted in revised form 29 June 2008

\begin{abstract}
Long fiber reinforced thermoplastics (LFT) were processed by in-line compounding equipment with a modified single screw extruder. A pulse stimulus response technique using PET spheres as the tracer was adopted to obtain residence time distribution (RTD) of extrusion compounding. RTD curves were fitted by the model based on the supposition that extrusion compounding was the combination of plug flow and mixed flow. Characteristic parameters of RTD model including $P$ the fraction of plug flow reactor (PFR) and $d$ the fraction of dead volume of continuous stirred tank reactor (CSTR) were used to associate with fiber degradation presented by fiber length and dispersion. The effects of screw speed, mixing length and channel depth on RTD curves, and characteristic parameters of RTD models as well as their effects on the fiber degradation were investigated. The influence of shear force with different screw speeds and variable channel depth on fiber degradation was studied and the main impetus of fiber degradation was also presented. The optimal process for obtaining the balance of fiber length and dispersion was presented.
\end{abstract}

Keywords: polymer composites, reinforcements, fiber degradation, RTD, LFT

\section{Introduction}

Fiber reinforced thermoplastics are widely used for the advantages of weight savings, low production costs, and freedom of design. Long fiber reinforced thermoplastics (LFT) have been confirmed to possess significant improvement in the properties of stiffness, strength, and toughness over their counterparts of short fiber reinforced thermoplastics. Because of excellent mechanical properties, low production cost and good environmental protection, LFT has been broadly applied in the automotive industry over the last decade [1-2].

Longer fiber length and better fiber dispersion are the key factors to obtain excellent mechanical properties of LFT. The instance is that longer fiber length is in favor of enhancing strength and tough- ness, and the improvement of fiber dispersion is beneficial to increase stiffness [3]. However, fiber length was reduced seriously by undergoing interaction between fiber-equipment, fiber-fiber, and fiber-matrix during extrusion compounding of LFT [4]. As to fiber degradation indicated by fiber length and dispersion, the reduction of fiber length had been investigated, but few studies were focusing on fiber dispersion so far [5]. There is a conflict of factors which influence fiber length and dispersion during extrusion compounding of LFT, and how to obtain the optimal balance of fiber length and dispersion is the purpose of this study.

In the mixing process of polymer system, residence time distribution (RTD) plays a significant role in determining mixing capacity as well as components

*Corresponding author, e-mail: zhuanghui@vip.citiz.net

(C) BME-PT and GTE 
uniformity [6-7]. The extrusion compounding of polymer reaction, immiscible blend and reinforced plastics had been investigated by RTD analysis.

Measurements of RTD in a single-screw extruder were carried out during experimental studies of controlled chemical degradation of polypropylene (PP). A radioactive tracer method was employed, and the effect of screw speed, temperature, and reaction on RTD curves were examined [8]. In an extruder, the effect of screw configuration on residence time and mixing efficiency was studied for an immiscible PA6/PP blend. RTD was used to indicate the total mixing efficiency, and its association with the dispersion of minor phase [9]. The mechanism of fiber fracture and methods to reduce it through process improvement and machine design in fiber reinforced composites were studied by Karthik Ramani et al. It is found that the residence time, fill-up, and the intensity of mixing during extrusion compounding have a predominant effect on fiber fracture [10].

RTD can be studied by the introduction of a pulse tracer or by introduction of a step change in the concentration [11]. Also RTD can be determined by an analysis of melt flow, but it is very complicated. An alternative analysis is the construction of a flow pattern with conceptual models. The most widely reported models include a combination of plug flow and mixed flow [12-13]. Mean residence time (MRT) was used for evaluating the extent of fiber fracture, but no paper was reported using the RTD model for the evaluations between processing condition, machine design and fiber fracture.

In-line compounding equipment modified with a single screw extruder was used for processing LFT. PP and glass fiber were added into the extruder from primary hopper and downstream hopper respectively. Three factors of screw speed, mixing length, and channel depth which affected RTD curves were studied. Two characteristic parameters of RTD model were used for characterizing RTD curves. The effects on fiber degradation presented by fiber length and fiber dispersion were studied. Fiber degradation was associated with two characteristic parameters of RTD model, and also with above three factors. The influence of shear force with different screw speeds and variable channel depth on fiber degradation was studied and the main impetus of fiber degradation was also presented. The optimal process for obtaining the bal- ance of fiber length and dispersion was the purpose of the study.

\section{Experiment}

\subsection{Materials}

Homo-polymer polypropylene (Y1600, Shanghai Petrochemical Co., Ltd., China) was used as matrix resin. Its melt flow index was $16.0 \mathrm{~g} / \mathrm{min}$ at $230^{\circ} \mathrm{C}$. Glass fiber of direct roving with filament diameter $17 \mu \mathrm{m}$ (GF362, Jushi Group Co., Ltd., China) was used as the reinforcement.

\subsection{Experimental condition}

A modified single screw extruder SJ-45B from Beijing Plastics Machinery Institute (screw diameter was $45 \mathrm{~mm}$ and length to diameter ratio was 30 ) was used for processing LFT. Free flow screw of three leads was used for the study of extrusion compounding shown in Figure 1. Three screws of different channel depth $\left(H_{1}=3 \mathrm{~mm}, H_{2}=6 \mathrm{~mm}\right.$ and $H_{3}=9 \mathrm{~mm}$ ) were used in the study. Channel depth of $6 \mathrm{~mm}$ would be used unless otherwise noted.

As shown in Figure 2, three hoppers were equipped on the extruder, and the length from the first and the second hopper to the exit was $500 \mathrm{~mm}$ $\left(L_{1}=500 \mathrm{~mm}\right)$ and $250 \mathrm{~mm}\left(L_{2}=250 \mathrm{~mm}\right)$ respectively. Mixing length was defined by the length from the first hopper or the second hopper to the exit. Unless otherwise noted, mixing length was $500 \mathrm{~mm}$. PP was added into the extruder from the primary hopper. Extrusion temperature was controlled between 200 and $220^{\circ} \mathrm{C}$. Glass fiber, at $30 \%$

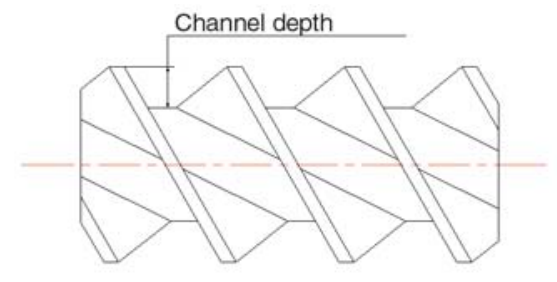

Figure 1. Schematic diagram of free flow screw

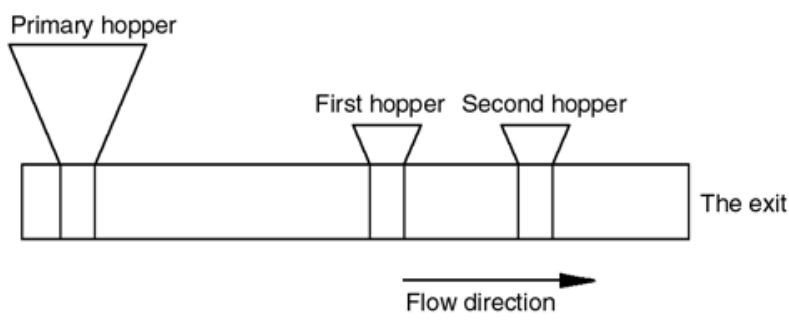

Figure 2. Diagram of the modified single screw extruder 
of both PP and glass fiber by weight, was added into the extruder from the first hopper or the second hopper. The extrudate was processed into plates of requested size by a calender, for analysis and testing purpose. Four screw speeds $\left(n_{1}=45.0 \mathrm{rpm}\right.$, $n_{2}=54.0 \mathrm{rpm}, n_{3}=67.5 \mathrm{rpm}$, and $n_{4}=81.0 \mathrm{rpm}$ ) were investigated.

\subsection{Fiber length and dispersion}

The fiber length measurements were conducted with a semi-image analyzer system. The matrix PP was removed by burning the plate off in a muffle furnace maintained at $500^{\circ} \mathrm{C}$ for $4 \mathrm{hr}$. The recovered glass fibers were dispersed in silicone-water suspension, and then the lengths of at least 800 fibers were measured by the semi-image analyzer system. Fiber length presented by number average $\left(L_{n}\right)$ was determined in Equation (1):

$$
L_{n}=\frac{\sum N_{i} L_{i}}{\sum N_{i}}
$$

Fiber dispersion is defined as the weight fraction of fiber filaments in total glass fiber. After the matrix PP was direct burnt in a muffle furnace maintained at $500^{\circ} \mathrm{C}$ for $4 \mathrm{hr}$, the weight of residual ash was defined as $W_{0}$, and fiber bundles, which were not dispersed into fiber filaments selected manually was defined as $W_{1}$. Fiber dispersion was determined in Equation (2):

$$
\text { Dispersion }=\frac{W_{0}-W_{1}}{W_{0}}
$$

\subsection{RTD test}

RTD curve was obtained using a pulse stimulus response technique, with black PET/poly (ethylene terephthalate) spheres as the tracer. 500 particles of the tracers were added into the extruder from the hopper that glass fiber had introduced, and the time was recorded simultaneously. The extruding plate of $100 \mathrm{~mm}$ width and $8 \mathrm{~mm}$ thickness was collected and black tracers in translucence PP can be seen clearly. The number of the tracers was counted at same time interval, and the fraction of the tracers in different time was obtained for RTD analysis. RTD curves were described as $F(\theta)$ versus $\theta$ curve.
Mathematically, these relations are described by Equations (3) and (4):

$$
\begin{gathered}
E(t)=\frac{C}{\int_{0}^{\infty} C \mathrm{~d} t}=\frac{C_{i}}{\sum C_{i} \Delta t_{i}} \\
F(t)=\int_{0}^{\infty} E(t) \mathrm{d} t=\frac{\sum_{i=0}^{t} C_{i} \Delta t}{\sum C_{i} \Delta t_{i}}
\end{gathered}
$$

where $C_{i}$ is the tracer concentration at time $t$.

The characteristic parameter of RTD curve, mean residence time (MRT) was calculates by Equation (5):

$$
M R T=\int_{0}^{\infty} t E(t) \mathrm{d} t=\frac{\sum t_{i} C_{i} \Delta t_{i}}{\sum C_{i} \Delta t_{i}}
$$

In order to compare RTD curves under different processing conditions, normalized time $\theta$ was defined by Equation (6):

$$
\theta=\frac{t}{M R T}
$$

and $F(\theta)$ was defined by Equation (7):

$$
F(\theta)=F(t)
$$

RTD curves of extrusion compounding at four screw speeds, two mixing length and three channel depth values have been measured. The analysis results of RTD curves were used to explain flow behavior of extrusion compounding, and more were associated with fiber degradation.

\subsection{RTD model}

The RTD curves were first modeled by considering extrusion compounding as the combination of mixed flow and plug flow by Wolf and Rescnick (1963). The model named Wolf model is expressed by Equation (8) [14]:

$$
\begin{aligned}
& F(\theta)=u(\theta)\left[1-e^{-\frac{\theta-P}{1-P}}\right] \\
& u(\theta)=0, \theta<P ; u(\theta)=1, \theta \geq p
\end{aligned}
$$

where $P$ is the fraction of PFR (plug flow reactor). 
The flow in CSTR was taken for mixed flow and the flow in PFR was considered as plug flow, so Yeh et al. introduces $d$, the fraction of dead volume in CSTR into Wolf model, so the model named Yeh model is expressed by Equation (9) [15]:

$$
\begin{aligned}
& F(\theta)=u(\theta)\left[1-e^{-\frac{\theta-P}{(1-d)(1-P)}}\right] \\
& u(\theta)=0, \theta<P ; u(\theta)=1, \theta \geq p
\end{aligned}
$$

The parameters $d$ and $P$ of above two models can be obtained by non-linear regression calculation, and were associated with fiber degradation.

\section{Results and discussion}

\subsection{RTD model}

RTD curve was shown as $F(\theta)$ versus $\theta$ for $81.0 \mathrm{rpm}$ screw speed and $6 \mathrm{~mm}$ channel depth in Figure 3. The RTD curve suggested that flow behavior was between plug flow and mixed flow. There was some declination between Wolf model

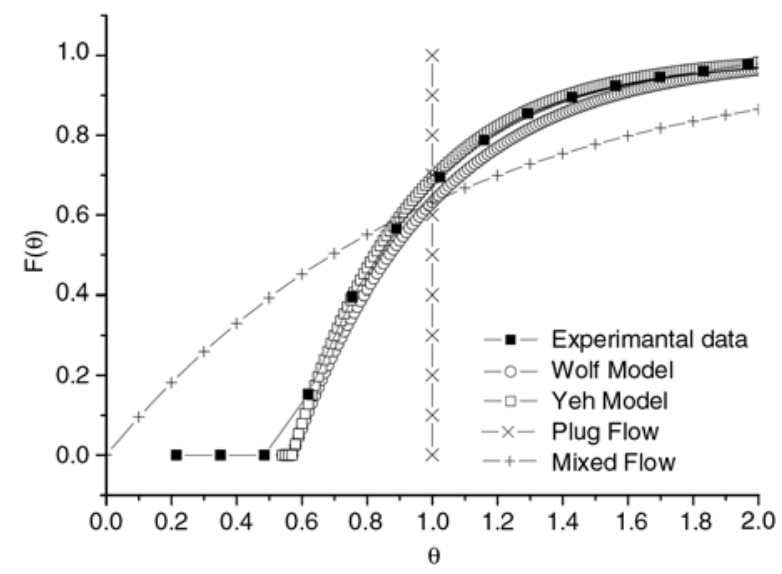

Figure 3. RTD curves as $F(\theta)$ versus $\theta$ with experimental data and different models and experimental data; but the consistency between Yeh model and experimental data was good.

Parameter values derived from Wolf model and Yeh model were listed in Table 1. In Yeh model, additional parameter $d$, the fraction of dead volume in CSTR was presented besides $P$, the fraction of PFR indicted in Wolf model. As shown in Figure 3, Yeh model was better than Wolf model for fitting experimental data. The existence of parameter $d$ indicated there was some flow-limited area during extrusion compounding.

\subsection{Influence of processing factors}

\subsubsection{Screw speed}

RTD curves with $6 \mathrm{~mm}$ channel depth was illustrated in Figure 4 by $F(\theta)$ versus $\theta$ for different screw speeds. It suggested that the flow behavior of extrusion compounding was between plug flow and mixed flow.

Parameters values derived from Yeh model at different screw speeds were listed in Table 2. The fraction of PFR $P$ and the fraction of dead volume

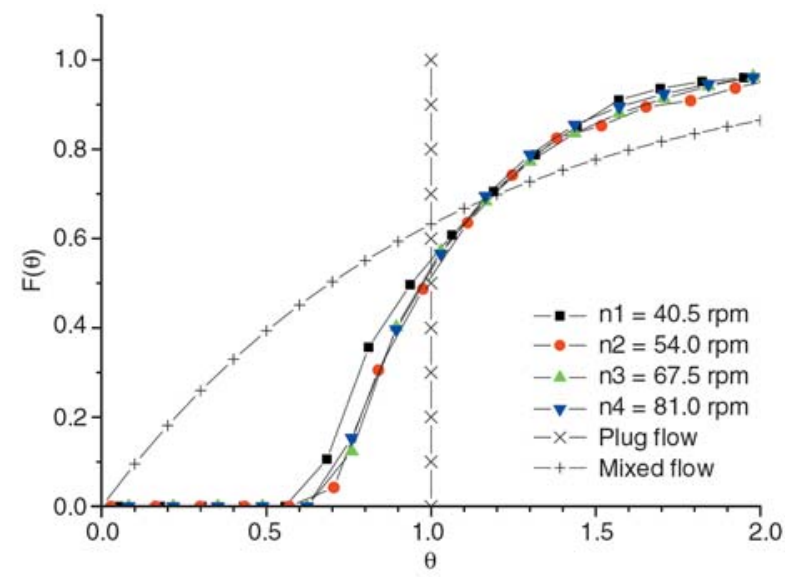

Figure 4. RTD curves as $F(\theta)$ versus $\theta$ at different screw speeds

Table 1. Parameter values derived from different models

\begin{tabular}{|c|c|c|c|c|c|}
\hline Screw No. & & Channel depth $[\mathbf{m m}]$ & Screw speed [rpm] & P & d \\
\hline S2 & Wolf model & 6 & 81.0 & 0.57 & - \\
\hline S2 & Yeh model & 6 & 81.0 & 0.57 & 0.15 \\
\hline
\end{tabular}

Table 2. Parameter values at different screw speeds

\begin{tabular}{|c|c|c|c|c|c|}
\hline Screw No. & Channel depth $[\mathbf{m m}]$ & Screw speed $[\mathbf{r p m}]$ & Mixing distance $[\mathbf{m m}]$ & P & d \\
\hline S2 & 6 & 40.5 & 500 & 0.56 & 0.14 \\
\hline S2 & 6 & 54.0 & 500 & 0.56 & 0.15 \\
\hline S2 & 6 & 67.5 & 500 & 0.56 & 0.15 \\
\hline S2 & 6 & 81.0 & 500 & 0.57 & 0.15 \\
\hline
\end{tabular}


Table 3. Fiber length and dispersion at different screw speeds

\begin{tabular}{|c|c|c|c|c|}
\hline Screw No. & Channel depth [mm] & Screw speed [rpm] & Fiber length [mm] & Fiber dispersion [\%] \\
\hline S2 & 6 & 40.5 & 12.78 & 30.58 \\
\hline S2 & 6 & 54.0 & 12.31 & 30.89 \\
\hline S2 & 6 & 67.5 & 11.82 & 31.54 \\
\hline S2 & 6 & 81.0 & 11.76 & 31.36 \\
\hline
\end{tabular}

in CSTR $d$ have no big variation at different screw speeds, which showed that the flow behaviors of extrusion compounding were almost the same. The results indicted that mixing capacity was slightly changed at different screw speeds.

Table 3 listed fiber length and dispersion at different screw speeds with $6 \mathrm{~mm}$ channel depth. As the screw speed increased from $40.5 \mathrm{rpm}$ to $81.0 \mathrm{rpm}$, the fiber length changed from $12.78 \mathrm{~mm}$ to $11.76 \mathrm{~mm}$, and the dispersion increased from 30.58 to $31.36 \%$. There were slightly change of fiber length and dispersion with increasing of screw speed. The results indicated that fiber length and dispersion would be somehow related to the value of $P$ and $d$.

\subsubsection{Mixing length}

Figure 5 listed RTD curves were presented by $F(\theta)$ and $\theta$ for different mixing length. RTD curves showed that flow behavior of extrusion compounding was between plug flow and mixed flow, and flow behavior of longer mixing length trended towards mixed flow.

Parameters values at different mixing length derived from Yeh model were listed in Table 4. The fraction of PFR P was decreased with the extension of mixing length, and the fact showed that the trend of the flow behavior was towards mixed flow. The fraction of dead volume in CSTR $d$ remained unchanged with the increment of mixing distance. Table 5 listed fiber length and dispersion with different mixing length at $81.0 \mathrm{rpm}$ screw speed and $6 \mathrm{~mm}$ channel depth. Fiber length was not changed

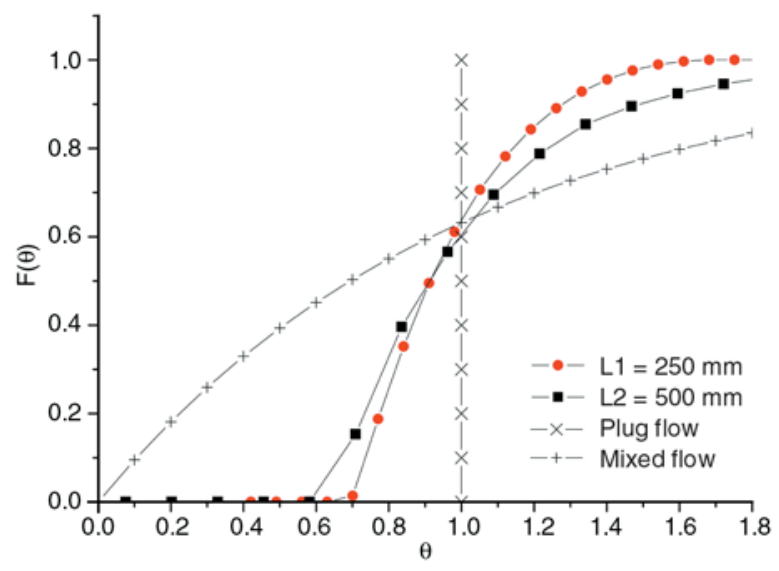

Figure 5. RTD curves as $F(\theta)$ versus $\theta$ at different mixing length

with the addition of mixing length, and fiber dispersion increased distinctly from 14.22 to $30.58 \%$.

As to Yeh model, the extruder is considered as the combination of PFR and CSTR, extrusion compounding is regarded as the combination of plug flow and mixed flow. The results showed that fiber dispersion was increased with the increment of $P$, and the increment of $P$ would impair fiber filament. Fiber length was not affected by the change of $P$, and the flow behavior of PFR, i.e. plug flow would not bring fiber to fracture severely.

\subsubsection{Channel depth}

\subsubsection{Mixing length of $250 \mathrm{~mm}$}

RTD curves presented as $F(\theta)$ versus $\theta$ were shown in Figure 6 at $250 \mathrm{~mm}$ mixing length, with different channel depth. It indicted that flow behavior of extrusion compounding was between plug flow and

Table 4. Parameter values at different mixing length

\begin{tabular}{|c|c|c|c|c|c|}
\hline Screw No. & Channel depth [mm] & Mixing length [mm] & Screw speed [rpm] & P & d \\
\hline S2 & 6 & 250 & 81.0 & 0.74 & 0.15 \\
\hline S2 & 6 & 500 & 81.0 & 0.57 & 0.15 \\
\hline
\end{tabular}

Table 5. Fiber length and dispersion at different mixing length

\begin{tabular}{|c|c|c|c|c|c|}
\hline Screw No. & Channel depth [mm] & Screw speed [rpm] & Mixing length [mm] & Fiber length [mm] & Fiber dispersion [\%] \\
\hline S3 & 9 & 81.0 & 250 & 12.84 & 14.22 \\
\hline S3 & 9 & 81.0 & 500 & 12.78 & 30.58 \\
\hline
\end{tabular}




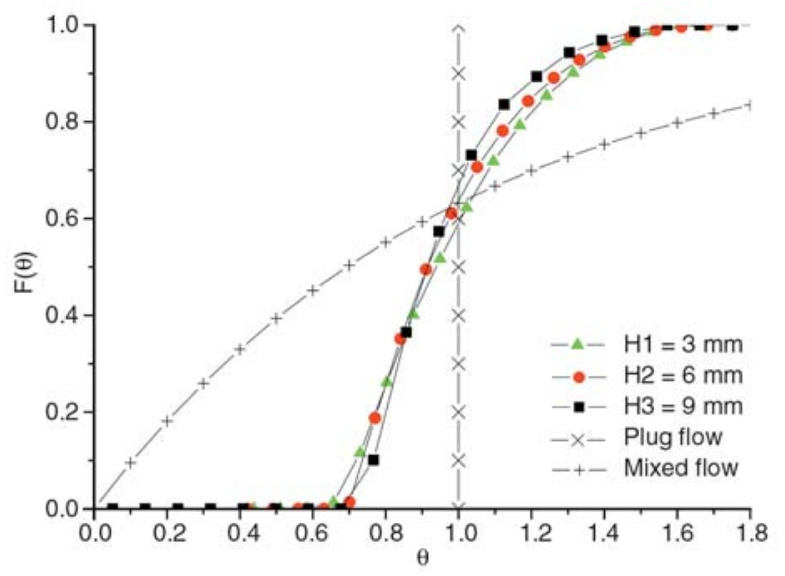

Figure 6. RTD curves as $F(\theta)$ versus $\theta$ at different channel depth for $250 \mathrm{~mm}$ mixing length

mixed flow, and flow behavior of bigger channel depth was close to plug flow.

Parameters values with different channel depth at $250 \mathrm{~mm}$ mixing length derived from Yeh model were listed in Table 6. The fraction of PFR $P$ was not changed with the addition of channel depth. The fraction of dead volume in CSTR $d$ was increased with the addition of channel depth, and the fact showed that flow behavior of bigger channel depth tended to plug flow.

Table 7 listed fiber length and dispersion with different channel depth and $250 \mathrm{~mm}$ mixing length. With the increasing of channel depth from 3 to $9 \mathrm{~mm}$, fiber length increased from 10.10 to $16.85 \mathrm{~mm}$ and fiber dispersion reduced from 21.04 to $7.42 \%$. The results showed that the increment of the fraction of dead volume in CSTR $d$ implied the improvement of fiber length and the impairment of fiber dispersion.

As to Yeh model, the extruder is considered as the combination of PFR and CSTR, extrusion compounding is considered to be a combination of plug flow and mixed flow. The mixing in a dead volume of CSTR is limited. With same value of $P$, the addi- tion of $d$ reduced mixing capacity of extrusion compounding. The variation of fiber length and dispersion was due to the reduction of mixing capacity presented by the increasing of the fraction of dead volume in CSTR $d$.

\subsubsection{Mixing length of $500 \mathrm{~mm}$}

RTD curves as $F(\theta)$ and $\theta$ with different channel depth at $500 \mathrm{~mm}$ mixing length were shown in Figure 7. RTD curves suggested that flow behavior of extrusion compounding was between plug flow and mixed flow. With the increasing of channel depth flow behavior approached to plug flow.

Parameter values with different channel depth at $500 \mathrm{~mm}$ mixing length derived from Yeh model were listed in Table 8 . The fraction of PFR $P$ was increased from 0.44 to 0.68 and showed that the flow behavior tended to plug flow with the increasing of channel depth. The fraction of dead volume in CSTR $d$ was increasing from -0.02 to 0.20 with the increasing of channel depth, which showed that the screw of deeper channel behaved with less mixing capacity. The value of $d(-0.02)$ for $\mathrm{S} 1$ screw

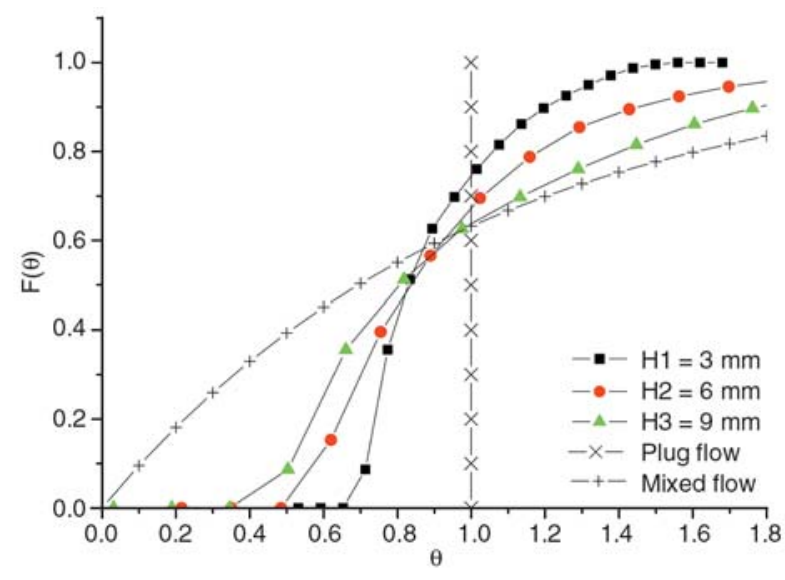

Figure 7. RTD curves as $F(\theta)$ versus $\theta$ at different channel depth for $500 \mathrm{~mm}$ mixing length

Table 6. Parameter values of $P$ and $d$ at different channel depth for $250 \mathrm{~mm}$ mixing length

\begin{tabular}{|c|c|c|c|c|}
\hline Screw No. & Channel depth [mm] & Screw speed [rpm] & P & d \\
\hline S1 & 3 & 81.0 & 0.76 & 0.04 \\
\hline S2 & 6 & 81.0 & 0.75 & 0.15 \\
\hline S3 & 9 & 81.0 & 0.74 & 0.29 \\
\hline
\end{tabular}

Table 7. Fiber length and dispersion at different channel depth for $250 \mathrm{~mm}$ mixing length

\begin{tabular}{|c|c|c|c|c|}
\hline Screw No. & Channel depth [mm] & Screw speed [rpm] & Fiber length [mm] & Fiber dispersion [\%] \\
\hline S1 & 3 & 81.0 & 10.10 & 21.04 \\
\hline S2 & 6 & 81.0 & 12.84 & 14.22 \\
\hline S3 & 9 & 81.0 & 16.85 & 7.42 \\
\hline
\end{tabular}


Table 8. Parameter values of $P$ and $d$ at different channel depth for $500 \mathrm{~mm}$ mixing length

\begin{tabular}{|c|c|c|c|c|}
\hline Screw No. & Channel depth [mm] & Screw speed [rpm] & P & d \\
\hline S1 & 3 & 81.0 & 0.44 & 0.02 \\
\hline S2 & 6 & 81.0 & 0.57 & 0.15 \\
\hline S3 & 9 & 81.0 & 0.68 & 0.21 \\
\hline
\end{tabular}

Table 9. Fiber length and dispersion at different channel depth for $500 \mathrm{~mm}$ mixing length

\begin{tabular}{|c|c|c|c|c|}
\hline Screw No. & Channel depth [mm] & Screw speed [rpm] & Fiber length [mm] & Fiber dispersion [\%] \\
\hline S1 & 3 & 81.0 & 7.61 & 65.64 \\
\hline S2 & 6 & 81.0 & 12.78 & 30.58 \\
\hline S3 & 9 & 81.0 & 14.74 & 24.44 \\
\hline
\end{tabular}

indicated that there existed severe back mixing besides forward mixing.

Table 9 listed fiber length and dispersion with different channel depths at $500 \mathrm{~mm}$ mixing length. Fiber length increased from 7.61 to $12.78 \mathrm{~mm}$ and fiber dispersion reduced from 65.64 to $30.58 \%$ with the increasing of channel depth. Those changes of fiber degradation were due to mutual effect of the addition of both $P$ and $d$.

\subsection{Influence of shear force}

In single screw extruder, shear rate could be expressed with constant channel depth and constant screw pitch by Equation (10) [16]:

$\dot{\gamma}=\frac{\pi \cdot D \cdot n}{H}$

where $\dot{\gamma}$ is simple shear rate, $D$ is screw diameter, $n$ is screw speed, and $H$ is channel depth.

Shear force brought by the melt under different screw speeds was listed in Table 10. When screw speed increased from 40.5 to $81.0 \mathrm{rpm}$, shear force rose from 63.72 to $124.8 \mathrm{~s}^{-1}$, glass degradation was not changed significantly based on the data listed in Table 3. Shear force brought by the melt under dif- ferent channel depth was shown in Table 11. Whereas channel depth increased from 3 to $9 \mathrm{~mm}$ with 250 and $500 \mathrm{~mm}$ mixing length, shear force rose from 62.4 to $187.2 \mathrm{~s}^{-1}$, glass degradation changed distinctly based on the data listed in Table 7 and Table 9. From the different changes of fiber degradation brought by the increasing of shear force, the it was concluded that shear force was not main impetus of fiber degradation.

In the process of LFT-PP extrusion, temperature range was during $200-220^{\circ} \mathrm{C}$, and corresponding $\eta$ was about $10^{5} \mathrm{~Pa} \cdot \mathrm{s}$, single shear rate was about $100 \mathrm{~s}^{-1}$ from the data in Table 10 and Table 11, see Table 8. So that the force $\dot{m}$ of glass fiber underwent during the process of LFT-PP extrusion would be about $10 \mathrm{M} \mathrm{Pa}$, as shown by Equation (11):

$(\dot{\gamma})=10^{2} \cdot 10^{5}=10^{7}$

Salinas et al. posted essential condition presented by Equation (12) that fiber would fracture during the compounding [17]. As for glass fiber, $\dot{\gamma}=$ $10^{6} \mathrm{MPa}$. The value of $\dot{m}$ that would bring glass fiber fracture is far more than that in the process of LFT-PP extrusion.

Table 10. Shear rate of S2 screw at different screw speeds

\begin{tabular}{|c|c|c|c|}
\hline Screw No. & Channel depth [mm] & Screw speed [rpm] & Simple shear rate $\left[\mathrm{s}^{-1}\right]$ \\
\hline S2 & 6 & 40.5 & 63.72 \\
\hline $\mathrm{S} 2$ & 6 & 54.0 & 83.20 \\
\hline S2 & 6 & 67.5 & 104.00 \\
\hline S2 & 6 & 81.0 & 124.80 \\
\hline
\end{tabular}

Table 11. Shear rate under different channel depth with $500 \mathrm{~mm}$ mixing dept

\begin{tabular}{|c|c|c|c|}
\hline Screw No. & Channel depth [mm] & Screw speed [rpm] & Simple shear rate $\left[\mathrm{s}^{-1}\right]$ \\
\hline S1 & 3 & 81.5 & 62.4 \\
\hline S2 & 6 & 81.5 & 124.8 \\
\hline S3 & 9 & 81.5 & 187.2 \\
\hline
\end{tabular}


$\dot{\gamma} \approx \frac{2 \sigma^{c}(\ln 2 r-1.75)}{r^{2}}$

As described by Wolf, during mixing glass fiber length was reduced by undergoing three interactions of fiber-equipment, fiber-fiber, and fiberpolymer [6]. Normally shear force of glass fiber applied by the melt would not bring glass fiber to be fractured because actual shear force resulted by the melt was far less than theoretical shear force derived by Salines as described above. But there are some regions such as the gap between screw edge and barrel or highly turbulent flow where fiber fracture would happen, the extent that brought glass fiber to fracture should be small. The main mechanism which brings glass fiber to facture would be fiber-equipment, and fiber-fiber interaction, and above two interactions would be improved by the enhancement of mixing capacity that could be presented by the characteristic parameters of RTD model such as $P$ and $d$.

\section{Conclusions}

Fiber degradation expressed by fiber fracture and fiber filament could be interpreted by characteristic parameters of RTD model such as the fraction of PFR $P$ and the fraction of dead volume in CSTR $d$. Fiber fracture characterized by fiber length was only increased with the increment of the fraction of dead volume in CSTR $d$. Fiber filament evaluated by fiber dispersion was impaired with the increasing of the fraction of PFR $P$ and the fraction of dead volume in CSTR $d$.

$P$ and $d$ were almost unaffected by the addition of screw speed, and there was a small reduction of fiber length and mild increment of fiber dispersion at same time. $P$ was decreased from 0.74 to 0.57 and $d$ showed no change with the addition of mixing length.There was a distinct increase of fiber dispersion from 14.22 to $30.58 \%$ and no change of fiber length with the increment of $P$.

With the increasing of channel depth from 3 to $9 \mathrm{~mm}$ for $250 \mathrm{~mm}$ mixing length, $P$ was almost same while there was significant increasing of $d$ from 0.04 to 0.29 . Fiber length was increased from 10.10 to $16.85 \mathrm{~mm}$ and fiber dispersion was decreased from 21.04 to $7.42 \%$ with the addition of $d$. With the increasing of channel depth from 3 to
$9 \mathrm{~mm}$ for $500 \mathrm{~mm}$ mixing length, $P$ and $\mathrm{d}$ showed significant change. $P$ was added from 0.44 to 0.68 and $d$ was increased from -0.02 to 0.21 respectively. Fiber length was increased from 7.61 to $14.74 \mathrm{~mm}$ and fiber dispersion was reduced from 65.64 to $24.44 \%$ with the increment of $P$ and $d$.

Screw speed and channel depth would bring some change of shear force. Shear force rise from 63.72 to $124.8 \mathrm{~s}^{-1}$ with screw speed increasing from 40.5 to $81.0 \mathrm{rpm}$, glass degradation was not changed significantly. Whereas shear force rise from 62.4 to $187.2 \mathrm{~s}^{-1}$ with channel depth increasing from 3 to $9 \mathrm{~mm}$, glass degradation was changed distinctly. The result was derived that shear force was not main impetus of fiber degradation.

Fiber degradation was brought by undergoing three interactions of fiber-equipment, fiber-fiber, and fiber-polymer during the extrusion. The main mechanism which brings glass fiber to facture would be fiber-equipment, and fiber-fiber interaction improved by the enhancement of mixing capacity that could be presented by the characteristic parameters of RTD model such as $P$ and $d$.

From the influence of process condition and screw configuration on fiber length and dispersion, $6 \mathrm{~mm}$ channel depth and $500 \mathrm{~mm}$ mixing length of free flow screw was appreciate for the optimal balance of fiber length and dispersion.

\section{References}

[1] Karian H. G.: Handbook of polypropylene and polypropylene composites. Marcel Dekker, New York (1999).

[2] Schemme M.: Long fibre reinforced thermoplastics. Kunststoffe, 93, 106-109 (2003).

[3] Bailey R., Kraft H.: A study of fibre attrition in the processing of long fibre reinforced thermoplastics. International Polymer Processing, 2, 94-101 (1987).

[4] Fisa B.: Mechanical degradation of glass fibers during compounding with polypropylene. Polymer Composites, 6, 232-241 (1985).

[5] Kuroda M. M. H., Scott C. E.: Initial dispersion mechanisms of chopped glass fiber in polystyrene. Polymer Composites, 23, 395-405 (2002).

[6] Wolf D., Holin N., White D. H.: Residence time distribution in a commercial twin-screw extruder. Polymer Engineering and Science, 26, 640-646 (1986).

[7] Lidor G., Tadmor Z.: Theoretical analysis of residence time distribution functions and strain distribution functions in plasticating screw extruders. Polymer Engineering and Science, 16, 450-462 (1976). 
[8] Tzoganakis C., Tang Y., Vlachopoulos J., Hamielec A. E.: Measurements of residence time distribution for the peroxide degradation of polypropylene in a singlescrew plasticating extruder. Journal of Applied Polymer Science, 37, 681-693 (1989).

[9] Vainio T. P., Harlin A., Seppälä J. V.: Screw optimization of a co-rotating twin-screw extruder for a binary immiscible blend. Polymer Engineering and Science, 35, 225-232 (1995).

[10] Ramani K., Bank D., Kraemer N.: Effect of screw design on fiber damage in extrusion compounding and composite properties. Polymer Composites, 16, 258266 (1995).

[11] Hornsby P. R., Singh D. P., Sothern G. R.: Determination of residence time distribution in polymer processing apparatus using tracer techniques. Polymer Testing, 5, 77-97 (1985).
[12] Yeh A-I., Jaw Y-M.: Modeling residence time distributions for single screw extrusion process. Journal of Food Engineering, 35, 211-232 (1998).

[13] Chen L., Pan Z., Hu G-H.: Residence time distribution in screw extruders. AIChE Journal, 39, 1455-1464 (1993).

[14] Wolf D., Rescnick W.: Residence time distributions in real system. Industrial and Engineering Chemistry Fundamentals, 2, 287-293 (1963).

[15] Yeh A. I., Jaw Y. M.: Modeling residence time distribution for single-screw extrusion process. Journal of Food Engineering, 35, 211-232 (1998).

[16] Booy M. L.: The influence of non-Newtonian flow on effective viscosity and channel efficiency in screw pumps. Polymer Engineering and Science, 21, 93-99 (1981).

[17] Salinas A., Pittman J. F. T.: Bending and breaking fibers in sheared suspensions. Polymer Engineering and Science, 21, 23-31 (1981). 